\title{
Expect the Unexpected: Bowel Obstruction Caused by Taenia saginata: A Case Report of Unusual Complications
}

\author{
Muhaned Alhassan, Ayman Ibraheim Barghash, Hatem Al-Saadi \\ Department of General Surgery, Suhar Hospital, Oman \\ Email: hondalhassan@gmail.com
}

How to cite this paper: Alhassan, M., Barghash, A.I. and Al-Saadi, H. (2021) Expect the Unexpected: Bowel Obstruction Caused by Taenia saginata: A Case Report of Unusual Complications. Case Reports in Clinical Medicine, 10, 191-196.

https://doi.org/10.4236/crcm.2021.107024

Received: May 4, 2021

Accepted: July 6, 2021

Published: July 9, 2021

Copyright ( 2021 by author(s) and Scientific Research Publishing Inc. This work is licensed under the Creative Commons Attribution International License (CC BY 4.0).

http://creativecommons.org/licenses/by/4.0/

(c) (i) Open Access

\begin{abstract}
Background: Small bowel obstruction (SBO) is a common gastrointestinal condition often warranting acute surgical intervention. In human, tapeworm species can cause a parasitic infection by ingestion of raw or under cooked beef ( $T$. saginata) or pork ( $T$. solium and $T$. asiatica). Taeniasis usually presents with vague symptoms or mild abdominal pain and discomfort. The intestinal complications are not commonly mentioned in the literatures. Case presentation: We present 65-year-old Egyptian woman who lives in Oman, who is known to have type two diabetes mellitus and hypertension. She presented to Emergency department with abdominal pain and constipation for a period of three days. On examination, she was dehydrated with tachycardia (pulse rate: 110/minute) blood pressure of $100 / 60 \mathrm{mmHg}$ and patient was afebrile. Abdominal examination revealed, mildly distended abdomen and tense, no guarding or rigidity no palpable mass, exaggerated bowel sound and rectum is empty on per rectal examination. Emergency laparotomy was performed with intra-operative findings of large foreign body impacted in the distal jejunum about $8 \times 3 \mathrm{~cm}$, cylinder in shape completely obliterating the lumen, distal loops was collapsed. Conclusion: Although Taeniasis is a very rare infection, index of suspicion such as a potential in the differential diagnosis as cause of an acute abdomen with unusual surgical complications should be considered. The diagnosis of these rare circumstances is usually made intraoperatively, and surgery is recommended only for the treatment of complications.
\end{abstract}

\section{Keywords}

Tapeworm, Cysticercus, Taenia saginata, Intestinal Obstruction, Proglottids 


\section{Introduction}

Small bowel obstruction (SBO) is a common gastrointestinal condition often warranting acute surgical intervention. In human, tapeworm species can cause a parasitic infection by ingestion of raw or under cooked beef ( $T$. saginata) or pork ( $T$. solium and T. asiatica). Althhough Taenia saginata can be found in many areas worldwide however, it is more prominent in tropical and underdeveloped countries [1]. It is associated with poverty, poor hygiene, and poor sanitations, therefore, health education, improvement in sanitation and hygiene play important part in prevention of such condition.

Taeniasis usually presents with vague symptoms or mild abdominal pain or discomfort. Symptoms can vary from less common symptoms like nausea, change in appetite, weakness or weight loss to more serious rare complications of intestinal obstruction and perforation of the gut [2]. The intestinal complications are not commonly mentioned in the literatures [2].

We are presenting a case of female patient presented with acute mechanical small bowel obstruction caused Taenia saginata infection as one of the rare causes of bowel obstruction.

\section{Case Presentation}

We present 65-year-old Egyptian woman who lives in Oman, who is known to have type two diabetes mellitus and hypertension. She presented to emergency department with abdominal pain and constipation for a period of three days. Pain was colicky in nature, sever started as a localized pain in the center of the abdomen then became diffuse to all over the abdomen. This was associated with nausea and constipation, but no vomiting or abdominal distention. She gave a history of attack of passing long white streaks like materials with her stool for one week before the development of her pain. However, no complains of bleeding per rectum or melena. No history of previous surgery, similar conditions nor weight loss.

On examination, she was dehydrated with tachycardia (pulse rate: 110/minute) blood pressure of 100/60 $\mathrm{mmHg}$ and patient was afebrile. Abdominal examination revealed, mildly distended abdomen and tense, no guarding or rigidity, no palpable mass, exaggerated bowel sound and rectum is empty on per rectal examination. Laboratory investigations showed WBCs of 11.70, neutrophil of 8.36, CRP: $329 \mathrm{mg} / \mathrm{L}$, and other blood investigations were normal. Abdomen X-ray showed dilated loops of small intestines with multiple air fluid levels. CT scan of the abdomen showed large segmental mildly enhancing circumferential thickening involve distal jejunum causing moderate narrowing of lumen and dilatation of proximal small bowel, feature suggestive of sub-acute Small bowel obstruction secondary to foreign body.

Patient was reviewed by anesthetic team and was consented for laparotomy. Emergency laparotomy was performed with intra-operative findings of large foreign body impacted in the distal jejunum about $8 \times 3 \mathrm{~cm}$, cylinder in shape 
completely obliterating the lumen, distal loops was collapsed. Resection of segment of affected small bowel was performed with primary anastomosis. No other intra-operative abnormalities were identified.

Post-operatively in recovery room, patient vomited large volume of bile containing large number of worms that were alive and moving. Patient was managed in recovery and was transferred to the ward. She recovered uneventfully and discharge home after 5 days of admission. She was then seen in outpatient clinic and was progressing well. Histopathology finding showed proglottid segments with eggs of taenia and adult worm.

\section{Discussion}

Taenia is a tapeworm acquired by ingesting undercooked beef or pork. Taeniasis is more common in Africa, Eastern Europe, Latin America and the Middle East. Most people with taeniasis will have no initial symptoms, however, as times goes, they will be aware of the infection as they pass proglottids in stool. Such gastric obstructive presentations are very uncommon in tapeworm infection, although enteric obstruction with subsequent gastric blockage has been previously reported. In contrast, bowel obstruction is a well-recognized complication of ascariasis [1].

Taenia species are common parasites that can infect humans. The two important species include the pork tapeworm or Taenia solium and the beef tapeworm or Taenia saginata. Taenia species found in Asia is a subspecies of Taenia saginata and it has been renamed as Taenia saginata asiatica. Taeniasis is endemic in Southeast Asia [2].

Humans are the definitive host. Adult tapeworms live in the human small intestine. Humans pass gravid eggs in feces; these mature eggs contaminate pastures and barnyards, where cattle and pigs ingest them. Upon reaching the alimentary canal of infected animals, the embryos are released, penetrate the gut wall, and enter the circulation. The embryos filter from the circulation and encyst in muscular tissue. Larvae (i.e., cysticerci) become infectious within 2 - 3 months. Humans develop a tapeworm infection by eating raw or undercooked beef or pork containing cysticerci. The cysticercus becomes activated, attaches to the wall of the small intestine by the scolex, and becomes a mature tapeworm. This maturation process takes 10 - 12 weeks for T. saginata and $5-12$ weeks for T. solium. A single tapeworm produces an average of 50,000 eggs per day and may live up to 25 years [3].

Examining the gravid proglottids helps identify the species; count the main uterine branches ( 7 - 16 branches for T. solium, 14 - 32 branches for T. saginata and 11 - 32 branches for T. asiatica) [4]. Examining the scolex helps differentiate the species because a $T$. solium scolex has 4 suckers and an armed rostellum and hooks but $T$. saginata scolex does not have rostellum and hook. T. asiatica has rostellum without a hook [4].

Histologic findings are mature cysticerci are ellipsoidal, translucent, flu- 
id-filled cysts, $1-2 \mathrm{~cm}$ in diameter. Younger cysticerci are smaller. A single dense white body can be seen through the membrane. The spiral canal of the cyst wall, which has a wavy appearance in most tissue preparations, is most frequently observed in biopsy specimens. The wall, which is $100-200$ micrometers wide, is characterized by an internal parenchymal layer of longitudinal and circular muscle, a middle layer of pseudoepithelial cells, and an outer cuticular layer composed of a dentate membrane with a microvillus projection that interfaces with host tissues. The scolex region is thickened and more organized. Cross sections of the scolex appear as several layers of folded smooth muscles, which may contain parts of the suckers or hooklets. The parasite is surrounded by an adventitia of host tissue reaction. A scant local cellular reaction that consists of some eosinophils and macrophages surrounds live cysticerci; dead cysticerci are surrounded by a dense inflammatory infiltrate that consists of the entire spectrum of inflammatory cells, including multinucleated giant macrophages [3].

Taenia saginata causes intestinal taeniasis, manifesting as abdominal discomfort, nausea, vomiting and weight loss. Some patients complain of passage of proglottids in the stool. Adult parasite may rarely be present in the stomach and the gastric secretions [3]. However, migrating proglottids have the tendency of causing bile duct inflammation, cholecystitis and appendicitis. Cases of intestinal small bowel perforation secondary to taeniasis have been reported in the literature.

Cysticercus attaches to the wall of the small intestine by means of scoleces and becomes a mature tapeworm. Intestinal taeniasis manifests as abdominal discomfort, indigestion, nausea, diarrhea, and weight loss [4]. Other complications include obstruction of bile ducts or pancreatic ducts, leading to cholecystitis, acute pancreatitis and granulomatous gastritis. The diagnosis of intestinal taeniasis depends on demonstration of the typical taenia eggs in the stool [5] [6].

Mechanical obstruction of small bowel is a very rare especially in unaffected communities. This indicates that taeniasis of the gastrointestinal tract can be the cause of the most unusual complications.

The diagnosis of these rare circumstances is usually made intraoperatively, and surgery is recommended only for the treatment of complications [6].

Individuals with intestinal Taenia infection might be asymptomatic or present with might symptoms. Once tapeworms are detected in faeces of infected individuals, anthelmintic therapy with praziquantel or niclosamide is usually sufficient. Such treatment could prevent cases of cysticercosis [7]. Asymptomatic cysticercosis requires no treatment. Cases with infection might be asymptomatic due to the long incubation period which could extends up to 16 years prior to symptoms become evident [8]. Asymptomatic cases with cysticercosis that are undiagnosed can have adverse outcomes if they were given albendazole or praziquantel as a treatment for other conditions. This is mainly caused by an inflammatory response from the dying parasite [7].

Treatment of such condition includes taking care of personal hygiene and ensuring meat and beef are cooked to avoid complications of teniasis. Along such 
lifestyle changes, medical treatment such as praziquantel or niclosamide can also be given [6]. Kalkan et al. 2013 have success removed Taenia endoscopically from the stomach [7]. Other endoscopic approaches that have been used are the injection of medication into small bowel wall allowing detachment of the tapeworms from the intestinal wall and execrated in stool.

\section{Conclusion}

Although Taeniasis is a very rare infection, index of suspicion such as a potential in the differential diagnosis as the cause of an acute abdomen with unusual surgical complications should be considered. The diagnosis of these rare circumstances is usually made intraoperatively, and surgery is recommended only for the treatment of complications. The possibility of parasitic infection should be kept in mind as a very rare possible cause of bowel obstruction even in non-endemic country and detailed history could helpful. Medical treatment of taeniasis includes oral medicine such as praziquantel or niclosamide, although prevention is the best to avoid serious complications of infection. However, in some cases endoscopic or surgical interventions might be needed.

\section{Conflicts of Interest}

The authors declare no conflicts of interest regarding the publication of this paper.

\section{References}

[1] Uysal, E. and Dokur, M. (2017) The Helminths Causing Surgical or Endoscopic Abdominal Intervention: A Review Article. Iranian Journal of Parasitology, 12 156-168.

[2] Fan, P.C., Lin, C.Y., Chen, C.C. and Chung, W.C. (1995) Morphological Description of Taenia saginata asiatica (Cyclophyllidea: Taeniidae) from Man in Asia. Journal of Helminthology, 69, 299-303. https://doi.org/10.1017/S0022149X00014863

[3] Uygur-Bayramiçli, O., Yavuzer, D., Dolapçioğlu, C., Sensu, S. and Tuncer, K. (1998) Granulomatous Gastritis Due to Taeniasis. Journal of Clinical Gastroenterology, 27, 351-352. https://doi.org/10.1097/00004836-199812000-00016

[4] Liu, Y.M., Bair, M.J., Chang, W.H., Lin, S.C. and Chan, Y.J. (2005) Acute Pancreatitis Caused by Tapeworm in the Biliary Tract. The American Journal of Tropical Medicine and Hygiene, 73, 377-380. https://doi.org/10.4269/ajtmh.2005.73.377

[5] Malik, A.A., Wani, R.A. and Bari, S. (2008) Acute Acalculous Cholecystitis Due to Taenia saginata. Annals of Saudi Medicine, 28, 388-389. https://doi.org/10.5144/0256-4947.2008.388

[6] Karanikas, I.D., Sakellaridis, T.E., Alexiou, C.P., Siaperas, P.A., Fotopoulos, A.C. and Antsaklis, G.I. (2006) Taenia saginata: A Rare Cause of Bowel Obstruction. Transactions of the Royal Society of Tropical Medicine and Hygiene, 101, 527-528. https://doi.org/10.1016/j.trstmh.2006.07.004

[7] Zammarchi, L., Bonati, M., Strohmeyer, M., et al. (2017) Screening, Diagnosis and Management of Human Cysticercosis and Taenia solium Taeniasis: Technical Recommendations by the COHEMI Project Study Group. Tropical Medicine \& Interna- 
tional Health, 22, 881-894. https://doi.org/10.1111/tmi.12887

[8] Nash, T.E., Ware, J.M. and Mahanty, S. (2018) Intraventricular Neurocysticercosis: Experience and Long-Term Outcome from a Tertiary Referral Center in the United States. The American Journal of Tropical Medicine and Hygiene, 98, 1755-1762. https://doi.org/10.4269/ajtmh.18-0085 